\title{
Análisis estratégico de la promoción inmobiliaria en España
}

\author{
Martín García, Rodrigo* \\ González Arias, Julio** \\ Mendoza Rivas, Manuel Ángel ${ }^{* * *}$
}

\section{Resumen}

El presente artículo analiza las oportunidades y dificultades presentes en las empresas de promoción inmobiliaria en España, sector que sufre una situación de desplome de demanda e importantes dificultades de financiación. Para ello, se empleó una doble metodología. En primer lugar, se definieron las variables críticas del negocio, según el Modelo de las Cinco Fuerzas Competitivas de Porter y, en segundo lugar, se realizó un benchmarking del sector para entender el comportamiento de sus principales protagonistas y así inferir en las líneas de actuación que marcarán el devenir de la promoción inmobiliaria. Además, se observó el proceso de bancarización como consecuencia de la refinanciación de la deuda y el exhaustivo análisis de riesgos de las operaciones y la importancia del aprovechamiento de las medidas públicas de apoyo al alquiler para la supervivencia empresarial. Los resultados evidencian que los productos sustitutivos de la nueva promoción tienen gran intensidad en el modelo, así como los propietarios de suelo, que absorben gran parte del valor. Competidores actuales y potenciales poseen menor fuerza, junto a los clientes, a pesar de encontrarse el negocio en un momento de baja demanda, por su fragmentación y heterogeneidad. Como conclusión se pone de manifiesto el necesario cambio de modelo hacia otro más sostenible, basado en la innovación y las nuevas tecnologías para recuperar el papel de pilar económico, que servirá de ejemplo en otros países.

Palabras clave: Promoción inmobiliaria, análisis de porter, bancarización, riesgo inmobiliario, benchmarking.

Recibido: 14-11-10. Aceptado: 21-04-11

* Profesor Doctor en Economía Financiera, Departamento de Economía de la Empresa y Contabilidad de la Universidad Nacional de Educación a Distancia (UNED).

E-mail: rmarting@cee.uned.es

** Profesor de Economía Financiera del Departamento de Economía de la Empresa y Contabilidad de la Universidad Nacional de Educación a Distancia (UNED). E-mail: jglez@cee.uned.es

*** Gerente de Planificación Financiera y Control de Gestión de Gerens Hill Gestión de Activos. E-mail: m.mendoza77@gmail.com 
Análisis estratégico de la promoción inmobiliaria en España

Martín García, Rodrigo; González Arias, Julio y Mendoza Rivas, Manuel Ángel

\title{
Strategic Analysis of Property Development in Spain
}

\begin{abstract}
This paper analyzes the opportunities and challenges faced by real estate development companies in Spain, a sector that has suffered a turnaround in recent years, due to slumping demand and significant financing difficulties. A dual approach has been used. First, critical variables and value drivers for the business have been defined using Porter's Five Competitive Forces Model. Secondly, the industry benchmark was studied in order to understand the behaviour of its main protagonists and, thereby, infer the lines of action that will shape the future of this business. Furthermore, bankarization, or access to banking services, as a consequence of debt refinancing, exhaustive risk analysis of operations, and the importance of taking advantage of public measures that support rental housing for corporate survival were noted. Results show that substitutive products in the new promotion have great intensity in the model, as do the land owners who absorb much of the value of the business. Current and potential competitors have less strength, together with the clients, despite low demand during the last three years, due to fragmentation and heterogeneity. Conclusions indicate the need to change the model for one that is more sustainable, based on innovation and new technologies, in order to restore real estate development as an economic pillar, which will be an example for other countries.
\end{abstract}

Key words: Real estate promotion, Porter's Five Competitive Forces, bankarization, real estate risk, benchmarking.

\section{Introducción}

La promoción inmobiliaria comprende el conjunto de actividades necesarias para la promoción de proyectos de construcción de edificios residenciales y no residenciales mediante la consecución de los medios financieros, técnicos y físicos necesarios para la realización de tales proyectos con vistas a su venta posterior excluyendo, expresamente, la promoción de obras de ingeniería civil, todas las actividades de arquitectura e ingeniería, los servicios de dirección de obras relacionados con proyectos de edificación y la construcción de edificios, como se indica en la Clasificación Nacional de Actividades Económicas (CNAE-2009), enmarcada dentro de un proceso internacional de revisión de las principales clasificaciones económicas de uso estadísti- co. La promoción inmobiliaria, que conforma el subgrupo CNAE 41.1, se integra así dentro de las actividades de construcción (grupo 41), al contrario que en el anterior sistema de clasificación (CNAE-93 Rev.1), en la que se integraba dentro del grupo 70 "Actividades Inmobiliarias", más concretamente, en el 70.1. "Actividades inmobiliarias por cuenta propia”.

En el presente trabajo, se realiza un análisis estratégico de la actividad de promoción inmobiliaria en el momento de mayor riesgo para el sector en varias décadas, ya que la oferta supera con creces a la demanda y la accesibilidad a la vivienda se ha visto reducida drásticamente por el aumento de los precios y las restricciones al crédito de las familias y el sector.

Para ello, en primer término, se aplicará el Modelo clásico de las Cinco Fuerzas propuesto por Michael Porter 
(1979a:139; 1979b: 214-227; 1980:29)., incluyendo un análisis de la estructura del sector, atendiendo al tamaño de los actores que intervienen, especialmente en base al número de asalariados que integran sus plantillas, a través del cual se estudia el efecto que sobre la rentabilidad del sector tienen las variables: competidores actuales, competidores potenciales, productos sustitutivos, proveedores y clientes. La respuesta de las principales empresas ha originado una fuerte concentración del ápice estratégico del sector, primero, y la bancarización posterior, como resultado de la difícil digestión de las políticas de adquisiciones fuertemente apalancadas, que se recoge en el apartado 3. Asimismo, se muestra la evolución de las compañías que son, sin duda, los "benchmarks" del negocio a nivel nacional y hasta hace poco, a nivel europeo (Metrovacesa, Reyal-Urbis e Inmobiliaria Colonial).

El trabajo concluye con una visión esquemática del modelo y describiendo las perspectivas para el sector a medio plazo, en base a las medidas públicas relacionadas con la vivienda, los planes estratégicos de las principales compañías y la evolución prevista de la demanda.

\section{Las cinco fuerzas competitivas en la promoción inmobiliaria}

El Modelo clásico de las Cinco Fuerzas de Porter agrupa en cinco bloques las fuerzas que delimitan el atractivo o potencial de una industria, ofreciendo una idea, tanto de las expectativas razonables de generación de beneficios por parte de las empresas del sector, como de las relaciones entre los agentes que intervienen en el mismo.

Constituye un marco teórico, basado en su artículo "How Competitive Forces Shape Strategy" (Porter, 1979a:139) centrado en el análisis del entorno empresarial a fin de determinar el posicionamiento estratégico y desarrollado en 1980 con "Competitive Strategy", con posteriores revisiones, actualizaciones y respuestas a sus detractores académicos. En conjunto, ofrece una base teórica y conceptual sólida para el desarrollo de estrategias de negocio. Según Porter (1991:97), la estructura del sector afecta a la sostenibilidad de los resultados de las empresas, mientras que la posición refleja la capacidad de la empresa para establecer una ventaja competitiva sobre sus rivales.

Conforma una de las dos vías para conseguir ventaja competitiva sostenible, frente al enfoque de los recursos y la búsqueda de eficiencia, siendo ambas no excluyentes en la iniciativa empresarial. En cualquier caso, el definido por Porter es un marco conceptual sistemático ampliamente respaldado en la comunidad académica y profesional.

Este apoyo puede estar condicionado a que supuso una nueva vía de trabajo que reunía, por primera vez dos disciplinas: la organización industrial y la estrategia (Aktouf, 2005:76), para proporcionar un enfoque global en la toma de decisiones estratégicas. En una entrevista al autor, publicada en 2002 (Argyres y McGahan, 2002:43) explicaba cómo tuvo que deshacerse de pensamiento económico clásico: "[...] supuestos ceteris paribus no funcionan. Los gerentes deben tener en cuenta, todo. [son necesarios] marcos conceptuales [...]" más adapta- 
Análisis estratégico de la promoción inmobiliaria en España

Martín García, Rodrigo; González Arias, Julio y Mendoza Rivas, Manuel Ángel

dos a la realidad, añadiendo: "[...] Necesitamos un enfoque más riguroso y sistemático con que analizar las industrias y la posición que toman las empresas en sus respectivas industrias [...]" (Argyres y McGahan, 2002: 43-44).

En cualquier caso, son muchos los autores que, partiendo del marco de Porter, han profundizado en el estudio de la estrategia, aunque los desacuerdos provienen más de la aplicación del mismo a nuevos mercados.

Así, Narayanan y Fahey (2005: 207-223), empleando el análisis de Toulmin a la propuesta de Porter, muestran la falta de validez de sus premisas institucionales en las economías emergentes. Shin (2001:169) muestra, no sólo las consecuencias derivadas de la nueva complejidad que supone la redefinición del marco de competencia, sino la reconfiguración de la industria que traen consigo los negocios electrónicos.

En cualquier caso, como defienden Spanos y Lioukas (2001:908), en este contexto, el desempeño de la empresa depende tanto de factores propios, como de la propia industria y, podemos añadir, sin duda, que los factores económicos generales y el entorno global son determinantes fundamentales. La estructura del sector está condicionada por las actividades de la empresa y el enriquecimiento paulatino de la cartera de negocios -inherente a las necesidades de crecimiento de las compañías- hace que la industria sea una realidad dinámica.

\subsection{Productos sustitutivos}

Existe un elevado grado de sustituibilidad entre inmuebles de nueva pro- moción -resultado de la actividad de estas compañías- y los inmuebles preexistentes, independientemente de la finalidad con que sean adquiridos, ya sea para su utilización directa, para arrendamiento o inversión. En este sentido, se ha seguido el criterio de no considerar el arrendamiento como sustitutivo de los inmuebles de nueva promoción en propiedad, ya que propiedad y arrendamiento constituyen modalidades de tenencia de los mismos inmuebles, más que una forma de sustituir el producto que proporcionan las promotoras inmobiliarias. De hecho, los inmuebles, nuevos o preexistentes pueden incorporarse a los mercados de compra-venta o alquiler y dicha consideración supondría duplicar una parte del stock (sin ir más lejos, los inmuebles adquiridos por entidades patrimonialistas y puestos a disposición de potenciales arrendatarios).

De hecho, el factor localización constituye una parte importante de todo aquello que se considera calidad de la vivienda aunque la vida útil de inmuebles antiguos pueda ser menor que la asociada a los de nueva promoción y su precio sea inferior, en igualdad de condiciones. En términos medios, desde el año 1995, el precio del metro cuadrado de la vivienda nueva es, aproximadamente, un $2 \%$ mayor que el de la vivienda de más de dos años de antigüedad, según datos del INE. En términos agregados, cuando se comparan inmuebles nuevos y antiguos en localizaciones similares y de características análogas, ambos proporcionan una utilización similar y pueden contar con un grado de sustituibilidad más que significativo, sin que ello evite las preferencias concretas de los potenciales adquirentes. Así, el 
mercado de segunda mano debe tener una ponderación notable en la elaboración de estrategias y en el análisis de nuevos proyectos, sobre todo en la determinación del mercado potencial.

\subsection{Proveedores}

Los más relevantes son las constructoras y los proveedores de suelo. Las primeras no tienen una fuerza destacable, salvo en proyectos que exijan una elevada capacitación técnica, poco habitual en el negocio inmobiliario. Además, no existe una vinculación promotor-constructor más allá de la finalización de cada contrato firmado, al ser cada proyecto independiente de los anteriores y posteriores.

Los propietarios de suelo sí tienen un alto poder de negociación, motivado por la exclusividad que aporta la localización fija del suelo, factor estratégico a la hora de determinar las decisiones de adquisición de promotoras y demandantes finales. Además, el volumen de suelo edificable depende del proceso de urbanización; entre 2002 y 2007 el ritmo de urbanización fue intenso y desembocó en un fuerte crecimiento en la disponibilidad de suelo edificable.

\subsection{El nivel de competencia actual}

Los competidores no son, a largo plazo, una fuerza relevante, ya que, a pesar del intenso proceso de concentración que ha experimentado el grupo de las mayores compañías, el negocio sigue siendo poco concentrado y existen multitud de compañías capaces de ofrecer un producto equivalente, derivado de las características del suelo y de la construcción. En la Tabla 1 se muestra la estructura del recién escindido subgrupo 41.1 CNAE, en la nueva clasificación $2009^{1}$, del resto de actividades relacionadas con el mundo inmobiliario, con el que mantiene idéntica estructura y que muestra los efectos de la fuerte contracción de la demanda desde mediados de 2007. Sin embargo, la madurez alcanzada durante los últimos años ha llevado a una fuerte contracción de la demanda que ha hecho que las promotoras hayan tenido que replantear su posicionamiento. Así, han reducido fuertemente el peso de su cartera en productos vacacionales, como son los ubicados en zonas turísticas de costa, y han sustituido la primera residencia libre en favor de la protegida. Además, un número elevado de ellas, que se encontraban integradas verticalmente, han externalizado, total o parcialmente, el proceso de construcción.

No existen, por tanto, destacables barreras de salida del negocio, ya que cada promoción concluye en sí misma. A pesar de que el promotor queda vinculado ante el comprador por los vicios visibles (1 año) y los no visibles (10 años) del inmueble objeto del contrato, estos compromisos se repercuten a quien ha ejecutado las obras, por lo que la única obligación del promotor es subsidiaria. El promotor puede, así, repetir contra la constructora los gastos incurridos como con-

1 INE, boletín estadístico online. Real Decreto 475/2007, de 28 de abril. 
Análisis estratégico de la promoción inmobiliaria en España

Martín García, Rodrigo; González Arias, Julio y Mendoza Rivas, Manuel Ángel

Tabla 1

Las empresas promotoras en España por asalariados $(2009,2008)$

\begin{tabular}{lcccc}
\hline $\begin{array}{l}\text { CNAE 1009-411 } \\
\text { Promoción inmobiliaria }\end{array}$ & $\mathbf{2 0 0 9}$ & $\mathbf{2 0 0 8}$ & $\begin{array}{c}\text { Incremento } \\
\text { (\%) }\end{array}$ & incremento \\
\hline De 1000 o más asalariados & 0 & 0 & - & - \\
De 500 a 999 asalariados & 3 & 6 & $-50,00 \%$ & -3 \\
De 200 a 499 asalariados & 13 & 19 & $-31,58 \%$ & -6 \\
De 100 a 199 asalariados & 32 & 40 & $-20,00 \%$ & -8 \\
De 50 a 99 asalariados & 63 & 79 & $-20,25 \%$ & -16 \\
De 20 a 49 asalariados & 300 & 355 & $-15,49 \%$ & -55 \\
De 10 a 19 asalariados & 653 & 800 & $-18,38 \%$ & -147 \\
De 6 a 9 asalariados & 1.512 & 1.918 & $-21,17 \%$ & -406 \\
De 3 a 5 asalariados & 4.783 & 5.291 & $-9,60 \%$ & -508 \\
De 1 a 2 asalariados & 23.355 & 26.066 & $-10,40 \%$ & -2711 \\
Sin asalariados & 71.211 & 71.801 & $-0,82 \%$ & -590 \\
Total & 101.925 & 106.375 & $-4,18 \%$ & -4450 \\
\hline
\end{tabular}

NOTA: únicamente se muestra la evolución de los dos últimos años por la reestructuración de la clasificación CNAE, que limita y distorsiona el estudio de series temporales más largas.

Fuente: INE (Directorio Central de Empresas).

secuencia de los vicios imputables a la misma. Una vez terminada la obra, no existen activos materiales que desinvertir, ya que los recursos humanos adscritos tienen vinculación al proyecto concreto $\mathrm{y}$, además, es el personal de la constructora el que ejecuta los trabajos.

La diferenciación del producto final no viene determinada por el trabajo de la empresa promotora sino por el estudio de arquitectura, los materiales incorporados y la constructora que ejecuta el proyecto $y$, en la construcción, al igual que en la promoción, son diversas las empresas capaces de hacer frente a los proyectos, salvo que requieran una complejidad técnica que incapacite a muchas compañias, hecho poco frecuente. No es necesario inmovilizar elevados recursos propios, pudiendo acudir al sistema financie- ro para conseguir financiación con garantía hipotecaria y vinculada al proyecto.

Sin embargo, la capacidad de absorción del mercado será limitada durante los próximos años, a pesar del intento de las políticas públicas de incentivar nuevos proyectos, especialmente los destinados a las viviendas en régimen de alquiler. Asimismo, el tamaño adquirido por el mercado hace que la importancia relativa de los proyectos protegidos frente a los de promoción libre sea muy reducida, aunque desde 2008 haya recuperado parte del peso perdido, contradiciendo informaciones anteriores, como el Informe de Fiscalización del Plan de Vivienda 1998-2001, que ponía de manifiesto la incapacidad de las políticas públicas para ser el revulsivo de un mercado amplio para los recursos públicos disponibles 
para las políticas de vivienda ${ }^{2}$. A este respecto, se entiende por viviendas de protección oficial o viviendas protegidas las así calificadas o declaradas por el órgano competente, que cumplan los requisitos establecidos en la normativa estatal que regula los planes de vivienda o los establecidos en normativa específica que regula sus propios planes de vivienda, para favorecer el acceso de los ciudadanos a la vivienda (Ministerio de Fomento, 2010a). El precio de venta está fijado por el organismo concedente de dicha categoría. Por otra parte, se entiende por vivienda libre toda vivienda que no se encuentra sujeta a ningún régimen de protección pública y puede ser transmitida sin restricción entre partes (Ministerio de Fomento, 2010b). El precio de venta lo determina el mercado.

Del mismo modo que ocurre con los proveedores, no existe vinculación estable con los clientes que pueda desembocar en compromisos de actividad a largo plazo, ya que, en diferentes casos, el producto de esta industria no es de adquisición recurrente. Cuando se trata de compañías, esta vinculación es mayor, pero aún así, no resulta digna de mención.

\subsection{Competidores potenciales}

La promoción inmobiliaria no se encuentra cerrada a la entrada de nuevos competidores, por inexistencia de barreras de entrada en forma de exigencias de capacitación técnica, fuertes necesidades de capital, políticas gubernamentales que limiten o controlen el acceso al mercado, entre otras.

De hecho, la ejecución masiva de las garantías hipotecarias de las promociones financiadas durante los últimos años ha llevado a una fuerte bancarización de la actividad inmobiliaria, especialmente la promoción, dado que la exposición directa con que cuenta la banca española al sector inmobiliario se sitúa en torno a los 60.000 millones de euros, sin incluir los riesgos de la cartera de créditos, que suponen alrededor de los 402.000 millones en financiación al sector constructor y de promoción residencial.

Las entidades financieras son fuertes competidores en el ámbito comercial, ya que pueden ofrecer distintas condiciones crediticias para adquirentes de inmuebles con financiación del $100 \%$ de la valoración y aplicación de menores

2 Según el Ministerio de Vivienda, en la última nota de prensa publicada sobre este tema (25 de marzo, 2010), el número de viviendas protegidas iniciadas entre octubre y diciembre de 2009 supone el $56,4 \%$ del total. Se observa el aumento en las viviendas libres iniciadas en el cuarto trimestre $(20,42 \%)$ y en las protegidas iniciadas $(58,33 \%)$. Las viviendas protegidas iniciadas en todo el año 2009 representan casi la mitad del total. En 2009 se iniciaron en total $80.230 \mathrm{vi}-$ viendas libres y se han terminado 356.555 . Las viviendas protegidas iniciadas a lo largo del año pasado son 79.054 y se ha concluido la construcción de 68.575. El número de aprobaciones definitivas de rehabilitación aumentó un 57,1\% en el cuarto trimestre y a lo largo de 2009 el número total ascendió a 42.774. (Http://www.vivienda.es/es/index.php?option=com_content\&task= view\&id=1477\&ltemid=178). 
Análisis estratégico de la promoción inmobiliaria en España

Martín García, Rodrigo; González Arias, Julio y Mendoza Rivas, Manuel Ángel

spreads, cuando éstos forman parte de la cartera de las entidades financieras.

Las entidades financieras se encuentran en la necesidad de reducir el peso de los inmuebles en los balances, ya que el Banco de España elevó a primeros de año la provisión que bancos y cajas de ahorros deben realizar sobre el valor de tasación de los activos inmobiliarios que hayan permanecido en su balance durante más de un año desde el 10\% al $20 \%$, primero en noviembre de 2009 y, después, a primeros de año, al 30\%, tratando de incentivar las ventas de estos activos inmobiliarios con cierta antigüedad en balance. Otra de las medidas impuestas por el regulador financiero a las entidades sometidas a su supervisión fue la obligación de provisionar el $25 \%$ de la deuda mantenida con grandes inmobiliarias altamente endeudadas y en notoria situación de inestable liquidez.

Pero, más aún, son muchas las entidades financieras que han iniciado los trámites para construir en los suelos que les han sido adjudicados tras la crisis inmobiliaria. La transformación de suelo supone para la banca una fuerte creación de valor a largo plazo y un mejor tratamiento contable de estos activos, lo que repercute significativamente en sus cuentas, aunque perjudica significativamente a las empresas promotoras.

\subsection{Clientes}

Los principales demandantes son las familias, para las cuales, las propiedades inmobiliarias suponen el $79 \%$ de sus activos (Banco de España, 2005a). Son muy heterogéneos y altamente fragmentados, por lo que no tienen capacidad de negociación de las condiciones contractuales, como ocurre cuando se encuentran fuertemente concentrados y no constituyen a largo plazo una fuerza que introduzca dificultades a las promotoras. Sin embargo, después de la fuerte contracción de la demanda, que ha llevado a una caída de las transacciones del $45,5 \%$, con un impacto desigual: en vivienda nueva, del $18,4 \%$ y en segunda mano, del $60 \%$, en 2009 respecto de 2004 (véase Gráfico 1). El Servicio de Estudios del BBVA, considera que, "... tras completar su ajuste, el sector inmobiliario español deberá converger hacia un tamaño más sostenible [...] alrededor de 400.000 unidades al año..." (BBVA, 2009:5), muy lejos del máximo histórico de 2006, con más de 920.000 viviendas visadas.

Una variable fundamental que determina el comportamiento del cliente es la política fiscal, dado que condiciona de manera significativa el esfuerzo de las familias para acceder a la vivienda, considerado uno de los factores clave de adquisición por el Banco de España (Banco de España, 2005b:63). El tratamiento con que cuenta, tradicionalmente, la vivienda es de incentivo al ahorro, dado que fomenta la adquisición frente al alquiler. Para el conjunto de oferentes de vivienda, promotores y propietarios de vivienda, la existencia de bonificaciones fiscales a la adquisición es muy positiva.

Los ahorros fiscales futuros estimados por los contribuyentes hacen que éstos estén dispuestos a pagar un precio superior en el momento de la adquisición, lo que podría llamarse "efecto de capitalización del ahorro fiscal" en el precio final. Sin embargo, para la actividad de promoción, las distintas medidas fiscales y sus 


\section{Gráfico 1 \\ Transacciones inmobiliarias de viviendas. Total nacional $(2004,2009)$}

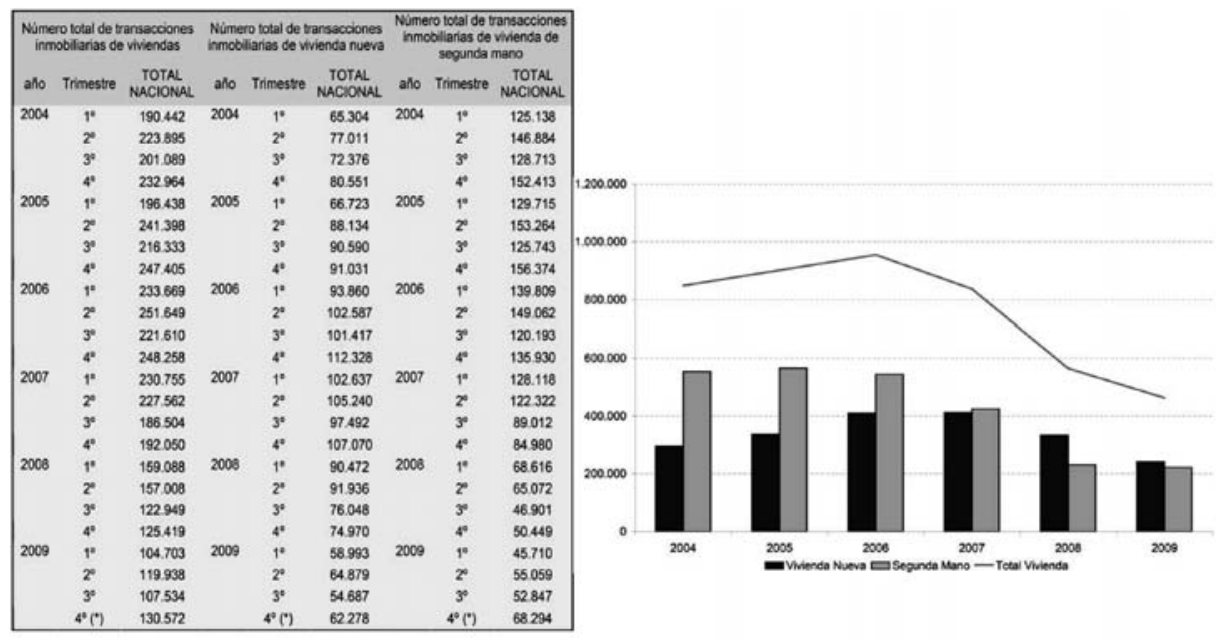

(*) Previsión.

Fuente: Ministerio de Vivienda.

efectos, así como el instrumento empleado para su puesta en funcionamiento, se recoge en el Diagrama 1. Es destacable el importante cambio que supondrá la nueva medida fiscal que elimina las deducciones por inversión en vivienda para todas las nuevas operaciones, lo que repercutirá negativamente en el volumen de demanda y tendrá efectos a todos los niveles en el mercado.

\section{Una Década de Contrastes: de la Expansión a la Contracción}

El notable incremento de demanda de vivienda en la primera mitad de década -motivado, entre otros, por la introducción del euro, la considerable disminución de los tipos de interés, la facilidad en la obtención de crédito, junto al notable alargamiento de los plazos de amortización, aderezado con factores demográficos, como el baby-boom, la demanda de extranjeros, vivienda vacacional e inmigración y otros (Taltavull, 2007:41)- provocó una intensificación en el esfuerzo inversor por parte de las empresas promotoras, con la compra de terrenos y solares, y en la actividad de producción del gremio, aceleración de trámites urbanísticos así como en la reducción de plazos de edificación y urbanización. Este momento fue aprovechado por algunos grupos no puramente inmobiliarios (Ferrovial, Iberdrola y Acciona, entre otros) para el abandono, parcial o total, del negocio inmobiliario, ante los elevados precios de las acciones del sector.

En los años 2006 y 2007, este aumento de la demanda produjo cierta exuberancia que originó movimientos en el 
Análisis estratégico de la promoción inmobiliaria en España

Martín García, Rodrigo; González Arias, Julio y Mendoza Rivas, Manuel Ángel

\section{Diagrama 1 \\ El impacto de las medidas fiscales sobre la vivienda}

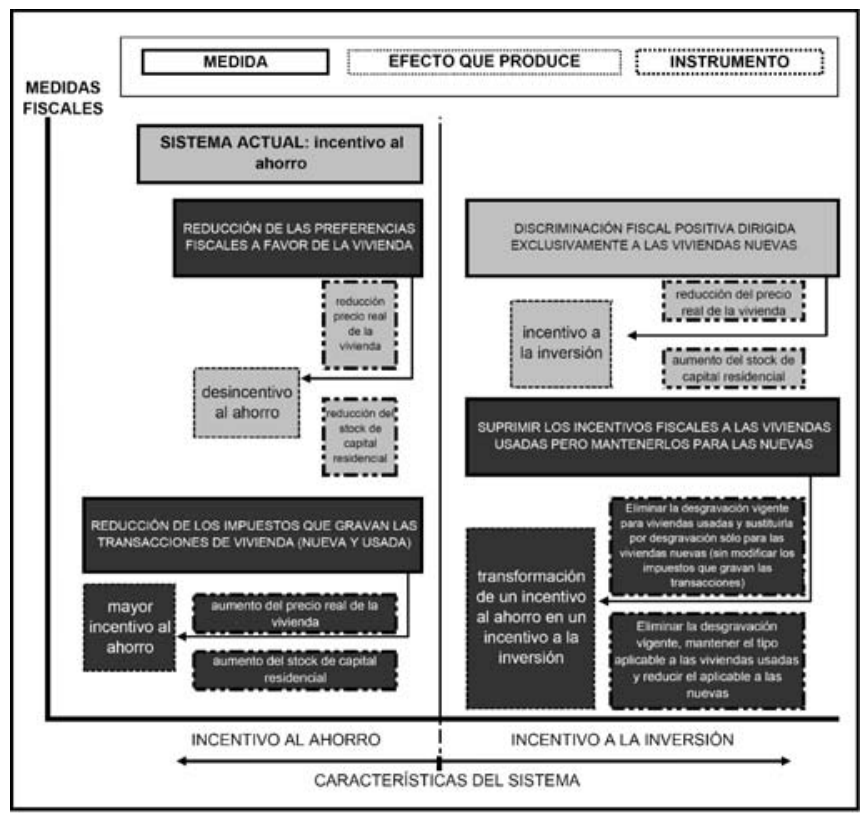

Fuente: Elaboración propia.

sector inmobiliario, produciéndose numerosas operaciones corporativas, desarroIlando estrategias muy agresivas con el fin de incorporar las carteras de suelo e inmuebles de sus competidores y, en algunos casos, de acceder a los mercados de valores. Estas operaciones corporativas son un instrumento rápido para conseguir carteras diversificadas de productos y mercados, y de internacionalizar su actividad. En esta actividad, para la que tan importante es la aplicación del precio de mercado, la valoración de estas empresas se realiza habitualmente en base al NAV -Net Asset Value- o Activo Neto Real, se pagaron sistemáticamente precios superiores a este valor, con elevado apalancamiento, a pesar del escaso re- corrido de los precios, como consecuencia del recalentamiento del sector y la subida de los tipos de interés.

Se recurrió a las salidas a bolsa por un lado, como instrumento de crecimiento y, por otro, para proporcionar liquidez a los accionistas de los proyectos originales, permitiendo a las empresas ampliar su base accionarial. En 2006 produjeron las salidas a Bolsa de Renta Corporación, Parquesol, Riofisa y Astroc y, en 2007, de Realia.

Una alternativa a la salida a bolsa, también empleada, fue la adquisición de empresas cotizadas, como hicieron Martinsa, Reyal o San José, con el objetivo de conseguir la máxima visibilidad en el mercado y la presencia de socios institucionales. 
A estas grandes tendencias se sumó el crecimiento internacional, especialmente en colaboración con socios locales, en países del este de Europa y Latinoamérica (México, Panamá, entre otros), y la consolidación del área patrimonial, ante una contracción de la promoción que ya comenzaba a esperarse (Seopan, 2008).

El fuerte retroceso de las operaciones, desde finales de 2007, ha producido un importante ajuste en el sector, en precios y demanda, que han llevado al deterioro de las perspectivas relativas al mercado inmobiliario. El elevado endeudamiento alcanzado por las inmobiliarias inmersas en este proceso de crecimiento ha modificado la percepción del riesgo de estas inversiones, obligando a la banca a endurecer las condiciones crediticias aplicadas a estas empresas. De este modo, se produjo un repunte de la aversión al riesgo en el sector, que se materializó en la reducción de la accesibilidad al crédito, la reevaluación del riesgo de las operaciones ya concedidas y el aumento de los spreads (diferenciales sobre el índice de referencia) aplicados. Esto supuso un importante revés para la política de expansión de estas compañías, tanto en su actividad corporativa, como en la de explotación. Desde mediados de junio de 2007 comenzaron a ser frecuentes las noticias relativas a la dificultad de sindicar los préstamos en este tipo de operaciones y los aumentos de tipos de interés de los mismos, además de reducciones en el periodo de devolución.

Así, llegó un momento en que las operaciones corporativas fueron suspendidas, ante el incremento de incertidum- bre: Tremón aplazó, primero y canceló después, su OPV por las duras exigencias aplicadas; Reyal-Urbis canceló su ampliación de capital.

A toda esta problemática sectorial, se sumaron nuevos factores externos con un fuerte impacto, tanto en la actividad ordinaria como la corporativa (Seopan, 2009):

- La crisis subprime, con un importante componente inmobiliario, que provocó la quiebra de Lehman Brothers y Bear Stearns con dos efectos inmediatos, dificultades en el acceso al crédito y subida de los tipos de interés. Ambos efectos penalizaron instantáneamente las transacciones y el precio de las mismas.

- El agotamiento del modelo económico en España, que redujo la demanda de vivienda y disparó el desempleo, principalmente en el sector de la construcción e industrias conexas. Según datos del INE, el número de parados en el sector de la construcción pasó de 294.100, en el primer cuatrimestre de 2008, a 601.500 en el mismo periodo de 2010.

Estos factores contribuyeron a una drástica reducción de la inversión inmobiliaria y de la demanda de vivienda, lo que produjo las primeras turbulencias en las principales empresas del sector, que venían de recientes operaciones de adquisición o fusión fuertemente apalancadas, con planes de negocios similares entre ellas, consistentes en la venta de suelo y promoción para centrar sus esfuerzos en incrementar su masa crítica en edificios en renta, que generasen ingresos recurrentes para cumplir con el servicio de las 
Análisis estratégico de la promoción inmobiliaria en España

Martín García, Rodrigo; González Arias, Julio y Mendoza Rivas, Manuel Ángel

cargas financieras, es decir, abonar los intereses derivados de las ingentes deudas acumuladas. Este fue el primer pilar de los planes de negocio que en general no se cumplió, pues al ser una estrategia común de los promotores, se pusieron a la venta numerosas bolsas de suelo, incluso en zonas cercanas entre sí, lo que aceleró el descenso del precio del suelo para hacer más atractivas determinadas bolsas de suelo ya que los posibles inversores podían elegir qué bolsa de suelo adquirir, previo acceso a la financiación por parte de las entidades financieras.

Al mismo tiempo, los principales accionistas, tras acaparar mediante OPA un alto porcentaje de participación en dichas sociedades, tenían previsto reducir sus participaciones mayoritarias mediante ofertas públicas de suscripción de acciones o ampliaciones de capital. Estas previsiones finalmente no pudieron ser llevadas a cabo debido a que los mercados financieros eran reacios a invertir más en el sector inmobiliario.

Al no poder hacer caja mediante ventas de suelo, ni poder financiarse con nuevos recursos propios o ajenos, los accionistas mayoritarios de estas empresas se vieron obligados a realizar ventas de inmuebles en renta, las conocidas "joyas de la corona", su única fuente de generación de ingresos recurrentes y de los que no deseaban desprenderse pues eran una de las claves de sus planes de negocio.

Aún así, estas empresas no pudieron cumplir con sus compromisos de pago de deuda, por lo que desde el año 2008 se han ido formalizando concursos de acreedores, numerosas refinanciaciones, incluso segundas refinanciaciones con tan solo dos ejercicios de diferencia respecto a la primera, daciones en pago de deuda, tanto con suelo e inmuebles o incluso en acciones, como en los casos de Metrovacesa y Colonial, donde la banca ha pasado de ser el principal acreedor a convertirse en el principal accionista de la empresa.

Estas operaciones se realizaron fuertemente apalancadas y han estado fundamentadas en valoraciones de activos realizadas por expertos independientes en la parte más alta del último ciclo inmobiliario en España, que ha sido el de mayores precios en la historia económica, con LTV (Loan To Value, relación deuda - valor de activos) del entorno del $50 \%$ al $65 \%$. Estas valoraciones se han visto notablemente reducidas desde entonces y no se evidencian previsiones de recuperación de las mismas a corto plazo, por lo que la LTV se ha visto incrementada al entorno del $70 \%$ al $80 \%$, de tal manera que las entidades financieras han visto decrecer sus garantías.

Se puede afirmar que la abultada deuda asumida por estas empresas y el incumplimiento de los planes de negocio trazados en los años de adquisiciones de empresas del sector, junto con el progresivo deterioro de las valoraciones de los activos adquiridos, han dañado notablemente su situación patrimonial, amenazando la solvencia.

Dado que las compañías que se sitúan en la cúspide de la pirámide aplican estrategias similares, su actuación tiene importantes repercusiones en el sector. Es lo que se denomina benchmarking de cartera, consistente en analizar los movimientos que realizan las empresas consideradas como referente, en la definición de su portafolio de negocios o áreas geo- 
gráficas de actuación, y la réplica de sus iniciativas, dado que se considera que la comparación con los líderes de la industria puede impulsar los resultados de aquellos que desarrollan esta técnica. Entre las compañías cuyo objeto social es la promoción inmobiliaria, destacan por su tamaño y liderazgo estratégico, las recogidas en el siguiente epígrafe, como benchmarks claros del negocio.

\section{Benchmarks de referencia}

La complejidad del estudio del sector inmobiliario/promotor se deriva de la multitud de interrelaciones directas e indirectas que se generan con otras empresas, que actúan en actividades complementarias o consecutivas, como construcción, comercialización y, en su caso, explotación por cuenta propia, así como las actividades inmobiliarias por cuenta de terceros. Este hecho, unido a la disparidad informativa, estratégica y competitiva existente entre los distintos segmentos de tamaño del sector y a la dificultad en la obtención de datos agregados suficientemente normalizados, complica el estudio y la obtención de conclusiones generales.

Incluso las empresas que están dentro del mismo segmento muestran estructuras organizativas muy diversas y la heterogénea publicación de información corporativa, tanto a nivel organizacional como de unidades de negocio, resta rigor al resultado de los análisis pormenorizados de rentabilidad y análisis de la antigüedad de la cartera, cuando la promoción se realiza sobre suelo procedente del propio activo, lo que complica la valoración de las sinergias y complica su contrastación objetiva.
Bajo este escenario, la metodología de estudio más adecuada es el Benchmarking (Intxaurburu y Ochoa, 2005:80), principalmente porque el comportamiento de las empresas de menor tamaño, habitualmente, reproduce las actuaciones de aquellas que se consideran de referencia. Por ello, el estudio de las compañías consideradas como benchmark mostrará el patrón que, con distintos retardos, aplicarán las empresas que componen la industria y, de forma general, la evolución paulatina de todo el sector.

Presentamos a continuación un análisis de las empresas inmobiliarias de referencia que, además de dar soporte al análisis contenido en el epígrafe anterior, servirá para establecer las perspectivas del sector, que quedarán recogidas en las conclusiones.

\subsection{Metrovacesa}

En el año 2002, fue adquirida por Bami, tras la compra del paquete de control que BBVA mantenía sobre Metrovacesa (23,9\% del capital), para un año más tarde fusionarse con ella, a fin de evitar nuevas ofertas hostiles sobre dicha compañía, como las ofertas públicas de adquisición de acciones realizadas en 2003 por Quarta y Astrid. Tras la mencionada fusión, Metrovacesa se convirtió en la mayor inmobiliaria europea con la compra a mediados de 2005 de la francesa Gecina y su posterior fusión, aportando una importante proyección internacional y reforzando el negocio del alquiler de inmuebles en su cartera.

Sin embargo, después de un año de intenso conflicto entre la familia Sa- 
Análisis estratégico de la promoción inmobiliaria en España

Martín García, Rodrigo; González Arias, Julio y Mendoza Rivas, Manuel Ángel

nahuja (39,6\% del capital social) y el equipo gestor (Joaquín Rivero respaldado por Bautista Soler, con un 33,1\%), ambas partes acordaron a finales de 2006 dividir el grupo valorado en unos 20.000 millones de euros, de forma que los Sanahuja pasaron a controlar la antigua Metrovacesa $(80 \%)$ y determinados activos franceses por valor de 1.800 millones de euros, mientras que la contraparte mantuvo la participación en Gecina y una parte de los activos en España, valorados en 450 millones. Este acuerdo de separación se formalizó a mediados de 2007 mediante la escisión de Gecina y Metrovacesa.

El endeudamiento neto fue creciendo con las sucesivas compras de sociedades hasta alcanzar un importe máximo de 9.478 millones de euros a diciembre de 2006. La escisión de Gecina durante 2007 , supuso una reducción del endeudamiento en unos 2.500 millones de euros y tras la venta de la torre de HSBC en Londres, descendió en otros $1.000 \mathrm{mi}-$ llones, con lo que la deuda a finales de 2008 era ligeramente superior a los 6.000 millones de euros, destacando el préstamo sindicado firmado para la compra de Gecina con un importe pendiente de unos 3.200 millones de euros. Aún considerando la notable reducción a dos tercios del endeudamiento máximo histórico en sólo dos ejercicios, a finales de diciembre de 2008 , la compañía solicitaba un "waiver", dispensa para el cumplimiento de las obligaciones financieras derivadas del préstamo sindicado, a los bancos mediante el cual se concedía una carencia de cuota de principal hasta 2011.

Los Sanahuja no fueron capaces de cumplir con los compromisos adquiridos en los procesos de adquisición y pos- terior escisión de Metrovacesa, por lo que los bancos financiadores optaron por la dación en pago de las deudas, convirtiéndose en los principales accionistas de la compañía (Santander, BBVA, Banesto, Banco Sabadell, Caja Madrid, Banco Popular, La Caixa y Barclays), con un $78 \%$ del capital social, mientras que los Sanahuja mantienen actualmente tan sólo un $17 \%$ del accionariado.

Una muestra del deterioro de la situación financiera e inmobiliaria es el patrimonio neto de la sociedad dominante del Grupo Metrovacesa, que asciende a 165,9 millones de euros a diciembre de 2009 , tras formalizar previamente la conversión en dos préstamos participativos de dos líneas de liquidez por un importe conjunto de 229 millones de euros (véase Tabla 2).

El reflejo contable de dicho deterioro en los dos últimos ejercicios cerrados lo encontramos en los epígrafes de "Gasto financiero neto sin capitalización de intereses" que ha supuesto un total de 650 millones de euros y "Deterioro de valor de activos" $y$ "Provisiones" que han ascendido a otros 550 millones de euros.

En la actualidad, Metrovacesa gestiona edificios en renta en España, Francia y Alemania y está intentando reducir su exposición al negocio promotor en España, con una agresiva campaña de descuentos en inmuebles.

\subsection{Reyal-Urbis}

Construcciones Reyal lanzó en julio de 2006 una OPA por el $100 \%$ de Inmobiliaria Urbis, que desembocó en la fusión entre ambas compañías, que salieron a cotizar conjuntamente en junio de 
Tabla 2

Principales Magnitudes de Metrovacesa

\begin{tabular}{|lcccc|}
\hline Ventas & 2006 & 2007 & 2008 & 2009 \\
- Alquiler & 771 & 257 & 281 & 199 \\
- Ventas de activos & 831 & 578 & 697 & 125 \\
- Promocion viviendas & 622 & 611 & 418 & 313 \\
- Suelo & 92 & 37 & 16 & 49 \\
- Servicios y otros & 10 & 8 & 9 & 13 \\
Ingresos totales & 2.326 & 1.492 & 1.421 & 699 \\
EBITDA & 980 & 471 & 226 & 117 \\
Gasto financiero neto sin capitalizacior & -224 & -341 & -431 & -216 \\
Capitalizacion & 52 & 50 & 60 & 28 \\
Gasto financiero neto con capitalizacior & -172 & -291 & -371 & -188 \\
Provisiones & -1 & -1 & -53 & -103 \\
Deterioro valor activos & - & - & -253 & -144 \\
Revalorizacion neta de inversiones de \\
negocios en \\
NuIF
\end{tabular}

Fuente: Memorias anuales de Metrovacesa (varios años).

2007. La sociedad fusionada es fundamentalmente promotora, con una gran cartera de suelo, y cuenta con un notable conjunto de edificios en renta, fundamentalmente oficinas y hoteles radicados en Madrid y Barcelona.

Dado el significativo volumen de deuda alcanzado a diciembre de 2006 (6.000 millones de euros), se plantearon distintas alternativas para la reducción paulatina del mismo, tales como la venta de grandes bolsas de suelo en zonas coincidentes en los bancos de suelos de las sociedades fusionadas, una Oferta Pública de Suscripción de acciones al público, mediante la disminución de la participación de control que Inveryal, la sociedad patrimonalista de Rafael Santamaría, tenía en Reyal Urbis, y la venta de las acciones de Colonial (6,4\% del capital social).

La situación de los mercados financieros desde mediados de 2007 ha sido desfavorable para la captación de recursos financieros en los mercados, lo que llevó a Reyal Urbis a descartar la posibilidad de realizar la OPS, por lo que centró sus esfuerzos en la venta de suelo (403 y 1.237 millones de euros en 2007 y 2008 , respectivamente) y en la venta de las acciones de Colonial, llevada a cabo en diciembre de 2007 por importe de 300 millones de euros a Nozar y Global Cartera de Valores, con cobro aplazado de una parte significativa de la venta. Esta cuenta a cobrar quedó saldada en julio de 2009 con Nozar, mediante canje por terrenos y la devolución de las acciones transmitidas inicialmente, mientras que Global Cartera de Valores solicitó concurso voluntario de acreedores el 4 de diciembre de 2008. Como conclusión, de las alternativas que se plantearon como vías de reducción del endeudamiento neto, sólo ha tenido resultados positivos 
Análisis estratégico de la promoción inmobiliaria en España

Martín García, Rodrigo; González Arias, Julio y Mendoza Rivas, Manuel Ángel

la venta de suelo, en gran parte a entidades financieras como dación en pago de deudas.

En octubre de 2008, tras la venta de suelos e inmuebles a entidades de crédito por un valor aproximado conjunto de unos 1.600 millones de euros, se alcanzó un acuerdo para la refinanciación de su crédito sindicado por importe de 3.000 millones de euros destinado a la compra de Urbis, con un alargamiento de los plazos de amortización, hasta el año 2015, y se facilitaron nuevas líneas de crédito como circulante, por un importe aproximado de unos 150 millones de euros.

En la actualidad y desde mediados de 2009, Reyal Urbis está negociando una propuesta de solución integral para la refinanciación de la deuda, unos 4.400 millones de euros, en la que se está considerando un periodo de carencia de principal e intereses hasta finales de 2012, con una reducción de inversiones corrientes y de gastos generales, que comprende un periodo de "hibernación" o paralización temporal de la actividad de promoción, salvo determinadas promociones en fase muy avanzada, y una línea de financiación puente para garantizar la ejecución del plan de negocio con un plan ordenado de ventas de activos de la sociedad. Previamente a la firma de la refinanciación, al igual que en el ejercicio 2008 , está previsto que se formalicen ventas de suelo por importe de unos 400 millones de euros a entidades financieras como dación en pago de deudas.

El reflejo contable del rápido deterioro financiero en los dos últimos ejercicios cerrados se encuentra en los epígrafes de "Gasto financiero neto", que ha supuesto un total de 605 millones de euros y
"Deterioro de valor de activos", que ha ascendido a otros 600 millones de euros, considerando el cambio de criterio seguido por Reyal Urbis durante 2009 de provisionar a valor neto realizable para existencias y valor en uso para inmovilizado, ya que de haber seguido manteniendo el criterio de deterioro de valor de activos en libros a valor de mercado, este importe se hubiera incrementado en unos 100 millones adicionales a los registrados (véase Tabla 3).

\subsection{Inmobiliaria Colonial}

La revolución en Colonial se inició con la OPA de Inmocaral sobre el $100 \%$ de Inmobiliaria Colonial en junio de 2006. Previamente, Luis Portillo, que sería presidente de Colonial hasta diciembre de 2007, había entrado en el capital de Inmocaral a finales de 2005, al adquirir el $72 \%$ de las acciones a La Caixa, con la intención de mantenerla en Bolsa, integrar los activos inmobiliarios de su sociedad Expo An y convertirla en una de las cinco empresas de referencia en el sector. Posteriormente, a inicios de 2007, Inmocaral-Colonial lanzó una OPA amistosa por el $100 \%$ de Riofisa, especializada en la promoción de centros comerciales y de ocio, que llevaba cotizando apenas 6 meses desde su salida a Bolsa en julio de 2006. Adicionalmente, la nueva Inmocaral-Colonial adquirió el 15\% de FCC en noviembre de 2006, de tal manera que su objetivo era tener ingresos por promoción y rentas procedentes de los activos procedentes de Colonial (oficinas) y Riofisa (centros comerciales) a los que sumar los dividendos que repartiera FCC. Previamente a esta adquisición de Colonial por 
Tabla 3

Principales Magnitudes de Reyal Urbis

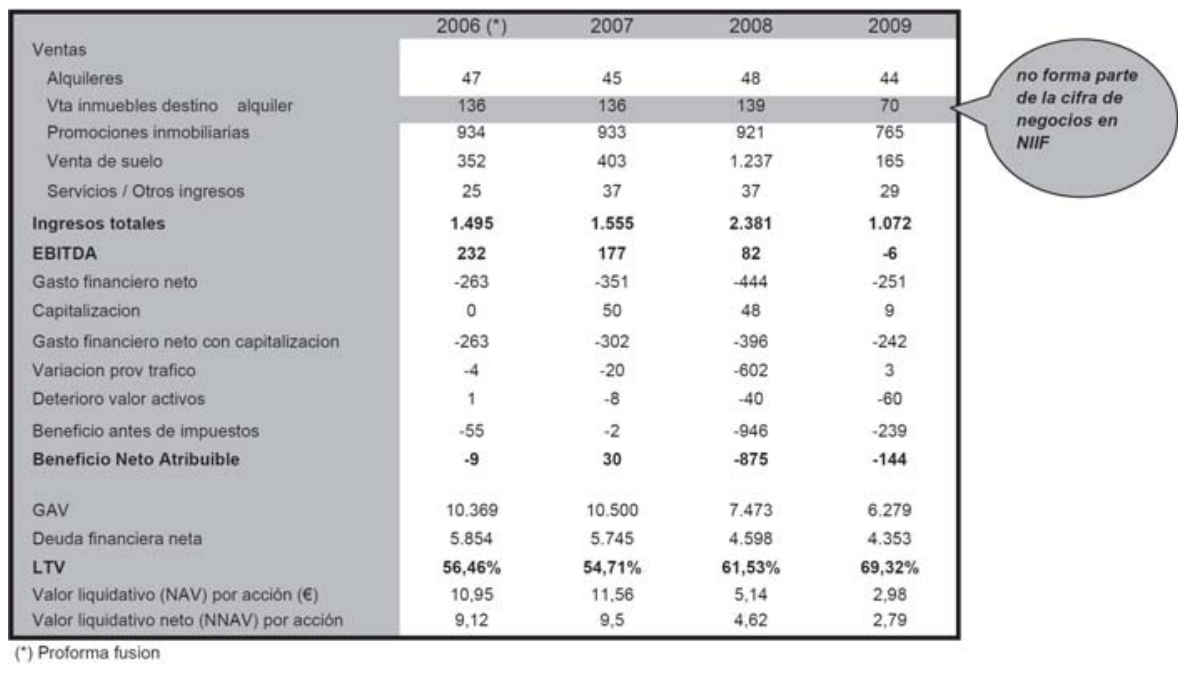

Fuente: Memorias anuales de Reyal Urbis (varios años).

Inmocaral, Colonial adquirió el control de la inmobiliaria gala SFL.

El peso de las deudas acumuladas por las sucesivas compras de sociedades, que alcanzó su máximo nivel en diciembre de 2007, con un importe cercano a los 9.000 millones de euros, y la disminución de los ingresos inicialmente previstos, han provocado las consiguientes ventas parciales de las participaciones en FCC y SFL. Estas ventas se han realizado mediante ejecución de opción de compra, otorgada a Calyon, del $3,4 \%$ de FCC y del $8,8 \%$ de SFL, por importe de unos 700 millones de euros, en enero de 2009, la venta de diversos activos de Colonial y Riofisa, así como la dación en pago de deudas en acciones para una parte de los bancos financiadores de los antiguos accionistas principales, Luis Portillo y Nozar, principalmente. Por otra parte, se ha producido un importante cambio en la composición del Consejo de Administración de la compañía, con la vuelta de Juan José Bruguera como presidente que, en la etapa anterior con la Caixa como principal accionista, fue su consejero delegado.

La primera dación en pago tuvo lugar en el primer semestre de 2008, convirtiéndose en accionistas, Banco Popular con un $9,1 \%$, La Caixa con un $5,4 \%$, y otras cinco entidades con un $9,35 \%$ en total, mediante la fórmula de sindicato de accionistas, con lo que les otorgaba un $23,85 \%$ del capital.

En septiembre de 2008, Colonial alcanzó un acuerdo para la reestructuración de su deuda, convirtiendo en deuda a largo plazo (5 años) un total de 6.500 millones de euros y mediante la emisión de bonos convertibles por un importe 
Análisis estratégico de la promoción inmobiliaria en España

Martín García, Rodrigo; González Arias, Julio y Mendoza Rivas, Manuel Ángel

aproximado de unos 1.400 millones, de los que fueron suscritos un total de 1.300 por los principales bancos acreedores y la venta de activos no estratégicos.

El cierre contable del ejercicio 2008 reflejó unos ajustes negativos de 1.970 millones de euros de la cartera inmobiliaria y de más de 2.300 millones por la participación en FCC y Riofisa, lo que repercutió directamente en el patrimonio neto de la compañía, que vio reducido sus fondos propios en unos 3.000 millones de euros en tan sólo un ejercicio. La emisión de bonos tenía como objetivo reforzar la solvencia patrimonial de Colonial y estabilizar financiera y operativamente a la compañía.

A finales de junio de 2009, Colonial suscribió un acuerdo de principios básicos de refinanciación con sus principales bancos acreedores (Calyon, RBS, Eurohypo y Goldman Sachs) que dejó en suspenso el cumplimiento de determina- das obligaciones contraídas en el préstamo sindicado, entre ellas, la venta de la participación que Colonial mantiene en Riofisa. Este acuerdo se ha formalizado el pasado 19 de febrero de 2010, reestructurando la totalidad de su deuda financiera:

1. Mediante la capitalización parcial de la deuda por ampliación de capital (3.900 millones).

2. Unos 2.100 millones de euros de deuda permanecerán vinculados a la actividad de gestión patrimonial, con nuevo vencimiento a 5 años.

3. Compromiso de conversión inmediata de los principales tenedores de bonos convertibles.

4. Segregación del negocio de suelo y promotor a una filial participada al $100 \%$ por Colonial.

Este acuerdo implica un notable refuerzo de la estructura de capital y una disminución de la deuda con motivo de la re-

Tabla 4

Principales Magnitudes de Inmobiliaria Colonial

\begin{tabular}{|c|c|c|c|c|c|}
\hline & 2006 & 2007 & 2008 & 2009 & \multirow{21}{*}{$\begin{array}{l}\text { no forma part } \\
\text { de la cifra de } \\
\text { negocios en } \\
\text { NIIF }\end{array}$} \\
\hline \multicolumn{5}{|l|}{ Ventas } & \\
\hline Alquileres & 76 & 301 & 289 & 282 & \\
\hline Vta inmuebles destino alquiler & 224 & 557 & 166 & 286 & \\
\hline Promociones inmobiliarias & 0 & 213 & 186 & 93 & \\
\hline Venta de suelo & 155 & 281 & 216 & 38 & \\
\hline Ingresos totales & 455 & 1.351 & 856 & 699 & \\
\hline EBITDA & 146 & 252 & 227 & 238 & \\
\hline Gasto financiero neto sin capitalizacion & -135 & -466 & -589 & -321 & \\
\hline Capitalizacion & 5 & 28 & 30 & 18 & \\
\hline Gasto financiero neto con capitalizacion & -130 & -438 & -559 & -303 & \\
\hline Amortizaciones y provisiones & 0 & -118 & -1.196 & 5 & \\
\hline Revalorizacion neta de inversiones inmobiliarias & 73 & 553 & -774 & -532 & \\
\hline Beneficio antes de impuestos & 17 & 265 & .2 .302 & .592 & \\
\hline Beneficio Neto Atribuible & 17 & 74 & -3.981 & -474 & \\
\hline $\mathrm{GAV}+\% \mathrm{FCC}$ & 10.498 & 13.214 & 9.255 & 7.074 & \\
\hline GAV & 8.964 & 11.630 & 8.930 & 7.038 & \\
\hline Deuda financiera neta & 6.785 & 8.961 & 7.349 & 4.545 & \\
\hline LTV & $64,63 \%$ & $67,81 \%$ & $79,41 \%$ & $64,25 \%$ & \\
\hline Valor liquidativo (NAV) por acción ( $€$ ) & 2,59 & 2,35 & 0,16 & 0.10 & \\
\hline Valor liquidativo neto (NNAV) por acción & 2,47 & 2,29 & 0.24 & 0,12 & \\
\hline
\end{tabular}

Fuente: Memorias anuales de Inmobiliaria Colonial (varios años). 
estructuración de unos 1.850 millones de euros, siendo a cierre de 2009 un total de 4.545 millones de euros (véase Tabla 4).

El deterioro de la situación patrimonial de la sociedad también ha sido motivado en parte por los gastos financieros derivados de la deuda, unos 1.400 millones de euros en los 3 últimos ejercicios (479 millones de euros en 2007, 589 en 2008 y 321 en 2009).

\section{Conclusiones}

El presente trabajo responde a una estructura de análisis similar al "TopDown" (Morales-Arce, 2004:556), justificada por las dificultades y limitaciones encontradas en el proceso de recopilación de datos -derivadas del reducido grupo de fuentes y su heterogeneidad, así como los cambios en los formatos y criterios de las series temporales, lo que complica notablemente la investigación, especialmente, por la diversidad de las empresas que intervienen en el mismo.

Cuestiones que no deben hacer decaer el ánimo de estudiar un sector que ha promovido importantes tasas de actividad económica, que ha constituido un importante motor de empleo y que durante unos años ha contribuido a mantener un crecimiento superior al de los países del entorno. Esta aportación pretende avanzar en el estudio de las empresas de referencia y en el conjunto de efectos que presentan las estrategias, políticas y procedimientos financieros empleados en el sector. A este respecto, debe destacarse la poca dedicación que, desde el ámbito investigador, se ha prestado a empresas con un potencial de arrastre tan fuerte sobre el conjunto de la economía.
La aplicación del marco conceptual de Porter al negocio de promoción inmobiliario, tal como quedó descrito en el apartado 2, destaca la intensidad de la fuerza ejercida por los productos sustitutivos de la nueva promoción, como son los inmuebles preexistentes, así como la intensidad de la fuerza que ejerce un grupo de proveedores, los propietarios de suelo, que absorben una parte muy relevante del valor creado por todo el negocio.

El retroceso de la actividad hace que ni competidores actuales ni potenciales sean las fuerzas de mayor intensidad y puede parecer contradictoria la reducida intensidad que caracteriza a los clientes en un momento de baja demanda como el actual, pero el elevado grado de fragmentación de que están dotados y su heterogeneidad restan poder de negociación cuando acuden al mercado.

Estas variables condicionan las expectativas del negocio y determinarán la evolución del contexto actual. Todos los datos apuntan a que la actividad de promoción inmobiliaria vivirá unos años de dificultades. El año 2010, que se proponía como el ejercicio de la recuperación, quedó condicionado, siguiendo al Banco de España por "[... ] una elevada tasa de paro, la pérdida acumulada en el valor de la riqueza patrimonial de las familias, en particular, de la vivienda, y las restricciones financieras a las que tienen que enfrentarse algunos hogares, lo que sugirió un descenso de la tasa de ahorro moderado y que se mantendrá en niveles superiores a su media histórica" (Banco de España, 2010).

Las previsiones a corto y medio plazo son la práctica paralización de desarrollos urbanísticos, bien por escasez de demanda de inmuebles y sobreoferta 
Análisis estratégico de la promoción inmobiliaria en España

Martín García, Rodrigo; González Arias, Julio y Mendoza Rivas, Manuel Ángel

en determinadas zonas o bien por problemas de tesorería de los propietarios de suelo. Este frenazo en seco de la actividad promotora irá acompañado de reducciones paulatinas de precios, para ir aligerando el stock acumulado. Simultáneamente, las compañías promotoras están reduciendo drásticamente sus costes generales de funcionamiento. El momento actual es el propicio para buscar el aplazamiento de deudas tributarias y la minimización de inversiones de cualquier tipo. Todo ello reforzará la especialización del sector, una vez que ya se ha producido la salida del negocio de los no especialistas.

Continuará el fuerte proceso de bancarización de la actividad inmobiliaria, tanto de inmuebles terminados, como en curso, incrementando la que ya es la más fuerte competencia contra los puros "role-players", especialmente proveniente de la asimetría en las condiciones crediticias aplicadas a adquirentes de inmuebles cuando éstos forman parte de la cartera de entidades financieras.

Las grandes compañías buscarán la refinanciación de la importante deuda corporativa tanto para las sociedades promotoras, como para las patrimonialistas, motivada por el incumplimiento de las obligaciones derivadas de operaciones societarias realizadas en los últimos ejercicios. La aplicación de fuertes covenants de deuda y compromisos vinculados a la gestión corporativa por parte de los bancos que financiaron las operaciones corporativas supone la fijación de importantes barreras a la gestión, acercándose a lo que podría denominarse como un sistema de "cogestión" o de "toma de control indirecta" de las sociedades inmobilia- rias, consecuencia de la dación en pago con acciones.

En adelante, las entidades financieras realizarán un exhaustivo análisis de riesgos de futuras operaciones de financiación, seguirán demandando equipos gestores solventes, condicionando su vinculación a la continuidad de aquellos a quienes han respaldado al mantener su presencia en las operaciones y programando la posible salida en el caso de que las pérdidas o la incertidumbre hagan necesario deshacer su posición.

Las empresas del sector deberán aprovechar las medidas públicas de apoyo al alquiler, especialmente con opción de compra para las compañías, como instrumento dinamizador de la cartera inmobiliaria (creación de las SOCIMI -Sociedades Cotizadas de Inversión en el Mercado Inmobiliario, nuevo vehículo de inversión con el que se pretende estimular el mercado de arrendamientos urbanos) (Morales-Arce, 2009; Congreso de los Diputados, 2009).

En definitiva, se observa un oscuro horizonte a medio plazo, ya que se ha producido la réplica de los planes estratégicos por parte de las inmobiliarias y ha llevado a que muchas de ellas busquen posiciones de liquidez a medio-largo y condicionen la renovación de créditos corporativos a plazos y líneas estratégicas equivalentes, mediante la venta de importantes bolsas de suelo, para ir atendiendo a sus compromisos financieros, y el incremento de la masa crítica de ingresos recurrentes.

En cualquier caso, y como ya viene reclamando desde hace varios trimestres ASPRIMA, la patronal madrileña del sector, el sector tenderá a reducir su peso, pero jugará un papel fundamental en el 
crecimiento económico de nuestro país (ASPRIMA, 2009:11). Este cambio de modelo implica virar el patrón de crecimiento hacia otro, más sostenible, en el que la innovación y las nuevas tecnologías cobren protagonismo.

A la vista del trabajo desarrollado y de las características de la actividad analizada, se plantea para futuras investigaciones, el interés de aplicar la perspectiva de los Recursos y Capacidades a la actividad de la promoción inmobiliaria, especialmente a dos fuentes primordiales de creación de valor en este negocio para los próximos años: a) todo aquello que se relaciona con la capacidad diferenciadora que proporcionan las características singulares del suelo a promover, y b) el potencial de marca en el segmento de mayor valor añadido del negocio: las viviendas nuevas de alta gama.

A modo de epílogo, se debe destacar de nuevo la importancia de la promoción inmobiliaria para la economía, más que en términos de valor añadido y el mercado laboral, en el efecto tirón sobre industrias conectadas y el impacto económico agregado. La burbuja inmobiliaria española, que acabó explotando coincidiendo con la última crisis financiera, debe ser analizada y se deben obtener enseñanzas claras y precisas, no sólo en el ámbito local, sino a nivel mundial, de cuáles son los peligros y actuaciones que deben ser sorteados, para evitar que la mala praxis contagie a toda la economía. El impacto de estas compañías es evidente y no sólo a nivel local o nacional, ya que aquellas que se han considerado benchmark del sector tienen posicionamiento en mercados internacionales.

\section{Referencias bibliográficas}

Aktouf, Omar (2005). "The False Expectations of Michael Porter's Strategic Management Framework". Revista Gestão e Planejamento, año $6, \mathrm{n}^{\circ} 11$, pags. 75-94.

Argyres, Nicholas; McGahan, Anita (2002). "An Interview With Michael Porter" Academy of Management Executive, vol. $16, n^{\circ} 2$, pags. 43-52.

ASPRIMA (2009). "Nuevo modelo para el sector inmobiliario español: la visión de Asprima". Revista trimestral ASPRIMA. $N^{\circ} 48$, págs. 8-11. Madrid.

Banco de España (2005a). "Efectos de la riqueza inmobiliaria sobre el consumo: resultados a partir de la Encuesta Financiera de las Familias". Boletín económico, mayo, págs. 55-62.

Banco de España (2005b). "Indicadores de accesibilidad y esfuerzo en el mercado de la vivienda". Boletín Económico, mayo, págs. 65-71.

Banco de España (2010). "Informe de proyecciones de la economía española". Boletín económico, marzo, págs. 3-15.

BBVA, Servicio de estudios (2009). Situación inmobiliaria. Segundo Semestre 2009. Madrid.

Congreso de los Diputados (2009). Ley $11 / 2009$ de 26 de Octubre por la que se regulan las Sociedades Anónimas Cotizadas de Inversión en el Mercado Inmobiliario (SOCIMI). BOE, 27 de Octubre.

Fundación de Estudios Inmobiliarios (1999). Libro Blanco del Sector Inmobiliario. Pamplona.

Gobierno de España (2007): Real Decreto 475/2007, de 13 de abril, por el que se aprueba la Clasificación Nacional de Actividades Económicas 2009 (CNAE-2009). BOE 102, de sábado 28 abril 2007. 
Análisis estratégico de la promoción inmobiliaria en España

Martín García, Rodrigo; González Arias, Julio y Mendoza Rivas, Manuel Ángel

Inmobiliaria Colonial (2010, 2009, 2008, 2007, 2006): Cuentas Anuales. Madrid.

Instituto Nacional de Estadística (INE). Directorio Central de Empresas (DIRCE) (http://www.ine.es/jaxi/menu.do?type $=$ pcaxis \&path $=\% 2 \mathrm{Ft} 37 / \mathrm{p} 201 \&$ file $=$ inebase\& $=0$ )

Instituto Nacional de Estadística (INE). Clasificación Nacional de actividades económicas, CNAE-2009 (http://www. ine.es/daco/daco42/clasificaciones/ cnae09/cnae2009.pdf)

Instituto Nacional de Estadística (INE). Correspondencias teóricas para fines estadísticos (http://www.ine.es/jaxi/menu. do?type $=$ pcaxis \&path $=\% 2 \mathrm{Ft} 40 \% 2$ Fclasrev\%2F\&file=inebase \&L=)

Intxaurburu, Miren; Ochoa, Carlos (2005). "Una Revisión Teórica de la Herramienta de Benchmarking", Revista de Dirección y Administración de Empresas, n 12, págs. 73-103.

Metrovacesa (2010, 2009, 2008, 2007, 2006). Cuentas Anuales. Madrid.

Ministerio de Vivienda (2010). Estadísticas e informes. http://www.mviv.es/es/.

Ministerio de Fomento (2010a). Estadística de Vivienda Protegida. Metodología. Subdirección General de Estudios y Estadísticas, Madrid, diciembre de 2010.

Ministerio de Fomento (2010b). Estadística de Vivienda Libre. Metodología. Subdirección General de Estudios y Estadísticas, Madrid, diciembre de 2010.

Morales-Arce, Rafael (2004). Teoría Económica de la Inversión. Ed. UNED.

Morales-Arce, Rafael (2009). La Ley 11/2009 que regula las Sociedades Anónimas Cotizadas de Inversión en el Mercado Inmobiliario (SOCIMI). Nota Técnica $n^{\circ}$ 10. Departamento de Finanzas. UNED, octubre.

Narayanan, V.; Fahey, Liam (2005). “The Relevance of the Institutional Underpin- nings of Porter's Five Forces Framework to Emerging Economies: An Epistemological Analysis" Journal of Management Studies vol. 42, $\mathrm{n}^{\circ} 1$, pags. 207-223.

Porter, Michael (1979a). "How Competitive Forces Shape Strategy," Harvard Business Review, Marzo-Abril, pags. 137-145.

Porter, Michael (1979b). "The Structure Within Industries and Companies Performance", Review of Economics and Statistics, 61(2), pags. 214-227.

Porter, Michael (1980). Competitive strategy: techniques for analyzing industries and competitors. Wiley. Nueva York.

Porter, Michael (1991). "Towards a Dynamic Theory of Strategy", Strategic Management Journal, 12 (Winter Special Issue), págs. 95-117.

Reyal-Urbis (2010, 2009, 2008, 2007). Cuentas Anuales. Madrid.

SEOPAN (2009, 2008, 2007, 2006). Informe anual de la construcción. Madrid

Servicio de Estudios del BBVA (2009). Situación Inmobiliaria, diciembre. Http:// serviciodeestudios.bbva.com

Shin, Namchul (2001). "Strategies for Competitive Advantage in Electronic Commerce" Journal of Electronic Commerce Research, vol. 2 , $n^{\circ} 4$, págs. 164-171.

Spanos, Yamir; Lioukas, Spyros (2001). "An examination into the causal logic of rent generation: contrasting Porter's competitive strategy framework and the resource-based perspective". Strategic Management Journal 22 (10), pags. 907-934.

Taltavull de la Paz, Paloma (2007). "La demanda de construcción en España: razones y tendencias recientes" Cuadernos de Información económica, $n^{\circ}$ 197, págs. 37-44. 